\title{
Complestao
}

\section{Reflexões sobre Arquitetura da Informação para dispositivos móveis}

\author{
Ítalo José Bastos Guimarães \\ Mestrando; Universidade Federal da Paraíba; adm.italoguimaraes@gmail.com \\ Marckson Roberto Ferreira de Sousa \\ Doutor; Universidade Federal da Paraíba; marckson.dci.ufpb@gmail.com
}

\begin{abstract}
Resumo: O artigo apresenta uma reflexão sobre a aplicação dos princípios da Arquitetura da Informação (AI) aos dispositivos móveis através de seu conceito e evolução tecnológica, da adequação segundo as recomendações feitas por Morville e Rosenfeld (2006) e Firtman (2013), e da ampliação da interação entre o homem e dispositivos. A metodologia adotada constituiu em um estudo exploratório através de levantamento bibliográfico que levou-nos a conclusão de que apesar das características particulares de cada dispositivo, é possível aplicar os sistemas de organização, rotulagem, navegação e busca, seja ele um smartphone, tablet ou e-reader (leitor de livros digitais). Cabe a nós, profissionais da informação, adaptarmo-nos a esta nova realidade e o desenvolvimento de aplicativos com base na arquitetura da informação com a finalidade de promover uma experiência satisfatória e agradável aos usuários, especialmente nas unidades informacionais.
\end{abstract}

Palavras-chave: Arquitetura da Informação. Dispositivos Móveis. Interação Humano-Dispositivo Móvel. Ambiente Informacional Digital.

\section{Introdução}

Os avanços tecnológicos vivenciados nas últimas décadas desafiam o campo da Ciência da Informação (CI) em suas diferentes unidades informacionais. Araújo (2014) apresenta a influência histórica das tecnologias para a CI e os impactos por elas causados, principalmente após o surgimento dos computadores, que possibilitaram a dissociação entre o suporte físico da informação e seu conteúdo, na conversão digital de livros, documentos e registros que poderiam assim ser melhor preservados e disseminados. 
Após o desenvolvimento do primeiro computador eletromecânico, as tecnologias continuaram influenciando as relações sociais e o modo como a informação era disponibilizada aos usuários. Por conta da evolução tecnológica gerada pelo crescente interesse desses usuários por computadores e pela internet, começaram a surgir as primeira dificuldades no acesso à informação e uso das tecnologias. Com a finalidade de sanar esses problemas, foram realizados os estudos iniciais sobre Arquitetura da Informação (AI). O termo foi disseminado pela primeira vez em 1976, por Richard Saul Wurman na Conferência realizada pelo Instituto Americano de Arquitetura, ainda que, em 1975, Wurman tenha escrito um artigo com Joel Katz denominado Beyond graphics: the architecture of information. Na década de 80, estudos eram conduzidos apenas tratando a AI como uma ferramenta para idealização e construção de computadores com ênfase na organização dos dados. Nos anos 90, autores como Morville e Rosenfeld, e Nielsen lançaram os primeiros livros sobre Arquitetura da Informação com ênfase na web (RESMINI; ROSATI, 2011).

Siqueira (2012) destaca a evolução do termo Arquitetura da Informação e seus variados conceitos apresentados por autores a partir dos anos 2000, como Hagedorn (2000), Dillon (2002), Haverty (2002), Bayle (2003), Lacerda (2005), Morvil-le e Rosenfeld (2006), Schiltz, Truyen e Coppens (2007), Costa (2010), Albuquerque (2010), Duarte (2010), Lima-Marques (2011) e Oliveira (2012). A partir de sua análise, constatou-se que é preciso adotar um padrão conceitual com a finalidade de minimizar os problemas causados na literatura devido aos inúmeros conceitos e suas diferentes interpretações.

No Brasil, há carência de pesquisas sobre AI, especialmente relacionando seus princípios aos dispositivos móveis, o que demonstra a relevância do presente trabalho para a comunidade científica. No período de 2003 a 2013, Silva e Souza (2014) realizaram um levantamento de produção científica no repositório BENAN-CIB - Questões em Rede (repositório que disponibiliza trabalhos e palestras dos Encontros Nacionais de Pesquisa e PósGraduação em Ciência da Informação - ENANCIBs) onde constataram a elaboração de apenas 100 artigos científicos em sua maioria em coautoria, que contemplavam temas como: arquitetura da informação; arquiteto da informação; 
arquitetura da informação pervasiva; e arquitetura de informação - sistemas de organização do conhecimento.

Neste artigo, buscamos conceituar os dispositivos móveis com a finalidade de apresentar aparelhos que possuem as características de mobilidade definidas por autores da Ciência da Computação, bem como aproximar os princípios da Arquitetura da Informação, aplicá-los à realidade dos dispositivos móveis, e discutir a ampliação da interatividade entre o homem e tais dispositivos, além de sua aplicação nas unidades informacionais. A metodologia da pesquisa constitui um estudo exploratório, visto que se pretende investigar o tema da adoção dos princípios de AI aos dispositivos móveis através do levantamento bibliográfico em livros e periódicos que abordem o assunto. Para tanto, realizou-se uma pesquisa na base de dados de periódicos da Capes com os termos: "information architecture", "mobile devices", "information architecture for mobile devices", "human commputer interaction" e outros termos correspondentes em língua portuguesa. Os livros selecionados estão relacionados aos dispositivos móveis e à arquitetura da informação por autores de áreas afins a CI. Devido à carência de livros e artigos que associam diretamente o tema estudado, e com a finalidade de cumprir o objetivo proposto, a intenção da pesquisa foi agrupar a base científica disponível sobre a AI e aplicá-la aos dispositivos móveis.

\section{Dispositivos móveis: conceito e evolução tecnológica}

No censo comum, imagina-se que qualquer produto tecnológico com possibilidade de locomoção pelos usuários pode ser considerado um dispositivo móvel. Entretanto, na literatura há definições mais apropriadas para o conceito, como quando Firtman (2013, p. 2, tradução nossa) destaca que para receber a denominação de dispositivo móvel, os aparelhos necessitam contemplar as características que seguem:

a) portáteis (portable) - pode ser utilizado em qualquer lugar pelos usuários; 
b) pessoal (personal) - precisa ser utilizado apenas para uso pessoal, onde o usuário define suas próprias características e personaliza conforme a sua necessidade;

c) companheiro (companion) - o dispositivo móvel passa a ser um companheiro do usuário, estando com ele a maior parte do tempo;

d) fácil de usar (easy to use) - precisa ser rápido e de fácil uso. O autor destaca que o notebook não se encaixaria nessa característica, pois o usuário precisa se sentar e procurar uma mesa para poder utilizá-lo, não se qualificando para o uso em qualquer ambiente e de modo rápido pelo usuário;

e) conectado (connected) - deve estar apto a se conectar com a internet quando o usuário precisar. É uma das justificativas adotadas por Firtman (2013) para afirmar que o notebook não se conceitua como um dispositivo móvel, pois precisa-se ligar o aparelho e aguardar a conexão com a internet.

Fitzek e Reichart (2007) apresentam três características que representam as funciona-lidades dos dispositivos móveis: a interface com o usuário - através dos diferentes tipos de entrada e saída de dados; a interface de comunicação, apresentado as di-ferentes formas de acessar a internet e trocar dados nos dispositivos móveis por meio de 3G, wi-fi e bluetooth; e os recursos internos, que se referem à estrutura adotada nos dispositivos como unidade de armazenamento, processamento central e bateria. Nesse sentido, Firtman (2013) afirma que os celulares (smartphones) que possuem conexão com a internet, os tablets e os e-readers (leitores de livros digitais) são con-siderados dispositivos móveis. No Quadro 1 é apresentada uma síntese das principais características defendidas por Firtman (2013), entre elas a diversidade no tamanho, a resolução e orientação da tela e os métodos de entrada. 
Quadro 1 - Características dos dispositivos móveis

\begin{tabular}{|l|l|}
\hline Característica & Dispositivo móvel \\
\hline Tamanho da tela & Geralmente menor que 4 polegadas. \\
\hline Resolução da tela & Bastante variada, não há um padrão exato adotado. \\
\hline Orientação da tela & Pode ser utilizada tanto de modo vertical quanto horizontal. \\
\hline Métodos de entrada & $\begin{array}{l}\text { Teclado numérico, teclado QWERTY, teclado virtual na tela sensível a } \\
\text { toque, reconhecimento de voz. }\end{array}$ \\
\hline
\end{tabular}

Fonte: Adaptado de Firtman (2013)

Quando tratamos da evolução tecnológica dos dispositivos móveis, é necessário recordar alguns fatos históricos que contribuíram para o avanço dos aparelhos: tudo começou em 3 de abril de 1974, quando o Motorola DynaTAC foi apresentado ao mundo. Após a fase de testes, o primeiro aparelho foi lançado em 1984 como DynaTAC 8000X com $33 \mathrm{~cm}$ de altura, 4,5 cm de largura e 8,9 cm de espessura. Em 1989, a Motorola lançou o segundo aparelho chamado de MicroTAC com um dispositivo analógico cujo captador de voz dobrava-se sobre o teclado - tecnologia conhecida como Telefone FLIP. Foi durante a década de 90 que surgiram as primeiras inovações como mensagens de texto e processadores de sinais e de alta tecnologia. Os celulares ofereciam aos usuários, além da realização de chamadas, a possibilidade de utilizarem agendas de endereços, calculadoras, pager e fax. No final da década de 90, a Nokia lançou o primeiro telefone em barra com display monocromático, Nokia 6160 com antena externa e bateria recarregável. Devido à relação custo versus benefício, o aparelho se tornou o mais vendido da década de 90. A partir dos anos 2000, o novo milênio trouxe as câmeras integradas, conexões com a internet e os smartphones. Atualmente, têm-se no mercado aparelhos modernos com tecnologia touchscreen, recursos que permitem a gravação de vídeos em alta definição, normalmente mais leves e finos (RENATO, 2012).

Além dos celulares, outros dispositivos móveis passaram por uma evolução tecnológica, como os tablets e os e-readers. O primeiro protótipo de tablet surgiu em 1968, quando Alan Kay lançou o Dynabook, um computador feito em um bloco único. Apenas em 1989, mais de 20 anos depois do protótipo, o primeiro tablet com características parecidas com as da atualidade foi lançado no mercado, denominado GridPad. Em 1992, a Microsoft lançou o TabletPC, 
mas devido ao custo elevado e poucas funcionalidades, não atraiu muitos consumidores. A popularização dos tablets ocorreu apenas em 2010 com o lançamento do iPad, produzido pela Apple (PINTO, 2014).

A história dos e-readers data de 1998, com o surgimento do Rocket ebooke Softbook. No ano seguinte, os livros começaram a ser comercializados em formato digital através de websites. A popularização do e-reader aconteceu a partir do aumento de interesse dos usuários pela leitura digital (AMOROSO, 2009).

O mercado dos dispositivos móveis sofre mudanças constantes e é por isso que é um desafio tentar prever o que poderá acontecer nos próximos anos. O que se pode afirmar com certeza é que essa tecnologia continuará a evoluir. Conforme ilustrado no Quadro 2, constata-se que cada aparelho possui suas especificidades técnicas, como sistema operacional, plataforma e navegador, que mudam de acordo com a empresa que o projeta.

Quadro 2 - Lista de Plataformas em Dispositivos Móveis

\begin{tabular}{|l|l|}
\hline \multicolumn{2}{|c|}{ Fabricantes/Plataformas } \\
\hline 1. Apple iOS & 8. HTC \\
\hline 2. Android & 9. HP webOS \\
\hline 3. RIM/BlackBerry & 10. Open webOS \\
\hline 4. Microsoft Windows Phone & 11. Barnes \& Noble Nook \\
\hline 5. Opera Mobile/Mini & 12. Tizen \\
\hline 6. Samsung Bada & 13. Firefox OS \\
\hline 7. Amazon Kindle & 14. Ubuntu for Phones \\
\hline
\end{tabular}

Fonte: Adaptado de Firtman (2013)

As diferentes plataformas, bem como os múltiplos contextos nos quais os dispositivos móveis são desenvolvidos, tornam a aplicação dos princípios da Arquitetura da Informação mais complexa e desafiadora. Entretanto, trata-se de uma realidade que precisa ser colocada em pauta, visto que, de acordo com Siqueira (2011), a previsão é que haja 55 bilhões de dispositivos móveis no mundo em 2020, isso significa que cada vez mais os smartphones, tablets e $e$ readers irão fazem parte do cotidiano da população mundial. 


\section{Arquitetura da Informação e sua aplicação para dispositivos móveis}

Estudos sobre a Arquitetura da Informação são aplicados aos ambientes informacionais desde a época de seu surgimento, com Wurman, na década de 70. São pesquisas desenvolvidas com a finalidade de descrever o papel e os desafios do profissional da informação, a aplicação da AI em diferentes contextos e as propostas de melhorias no uso da Arquitetura da Informação.

A partir da evolução do conceito e da relevância da AI para ambientes informacionais, pesquisadores desenvolveram importantes estudos sobre a temática na década de 90, como Wurman e Bradford (1996) com o livro Information Architects, Morville e Rosenfeld (1998) através da primeira edição do livro Information Architecture for the World Wide Web onde aplicaram os princípios da AI ao ambiente digital da web e Reiss (2000) no livro chamado Practical information architecture: a hands-on approach to structuring successful sites.

Dillon (2002) destaca que o objetivo da Arquitetura da Informação é realizar o processo de estruturação, implementação e avaliação dos espaços informacionais onde ocorre interação social. Os dispositivos móveis devem ser projetados com a finalidade de oferecer o melhor caminho ao usuário, ou seja, a informação deve ser organizada visando facilidade de uso e de aprendizado, além de promover o acesso rápido no processo de busca por informações.

Barker (2005) defende que o termo Arquitetura da Informação é utilizado para descrever a estrutura de um sistema, a maneira como as informações são agrupadas, os métodos de navegação e as terminologias utilizadas no sistema. Na literatura, há uma associação do termo à web devido à relevância dos estudos nessa área, porém a AI também pode ser utilizada em outros contextos digitais onde haja necessidade de se estruturar a informação, inclusive nos dispositivos móveis. Morville e Rosenfeld (2006, p.4, tradução nossa) definiram AI para web como:

a) projeto estrutural de ambientes onde a informação é compartilhada;

b) a combinação de organização, rotulagem, busca e sistemas de navegação em sites e Intranets; 
c) a arte e ciência de dar forma a produtos de informação e experiências a fim de apoiar a usabilidade e a facilidade de localização;

d) uma disciplina emergente de práticas focadas em trazer princípios do design e da arquitetura para o ambiente digital.

O Information Architecture Institute ampliou o conceito apresentado por Morville e Rosenfeld (2006) de modo que este contemplasse qualquer ambiente digital informacional, definindo-a como "[...] a arte e a ciência de organizar e catalogar websites, intranets, comunidades online e software de modo que garantisse a usabilidade" (INFORMATION ARCHITECTURE INSTITUTE, 2013, p. 1, tradução nossa).

A Arquitetura da Informação “[...] não cria somente estruturas de navegação para sites, também integra as necessidades dos usuários como exigências. Atua como defensora dos usuários nos ambientes informacionais digitais" (EWING; MAGNU-SON; SCHANG, 2002, p. 5, tradução nossa). Morville e Rosenfeld (2006, p. 25) apresentam três pilares da Arquitetura da Informação, ilustrados na Figura, a saber: contexto, conteúdo e usuários.

Figura 1: Os três pilares da Arquitetura da Informação

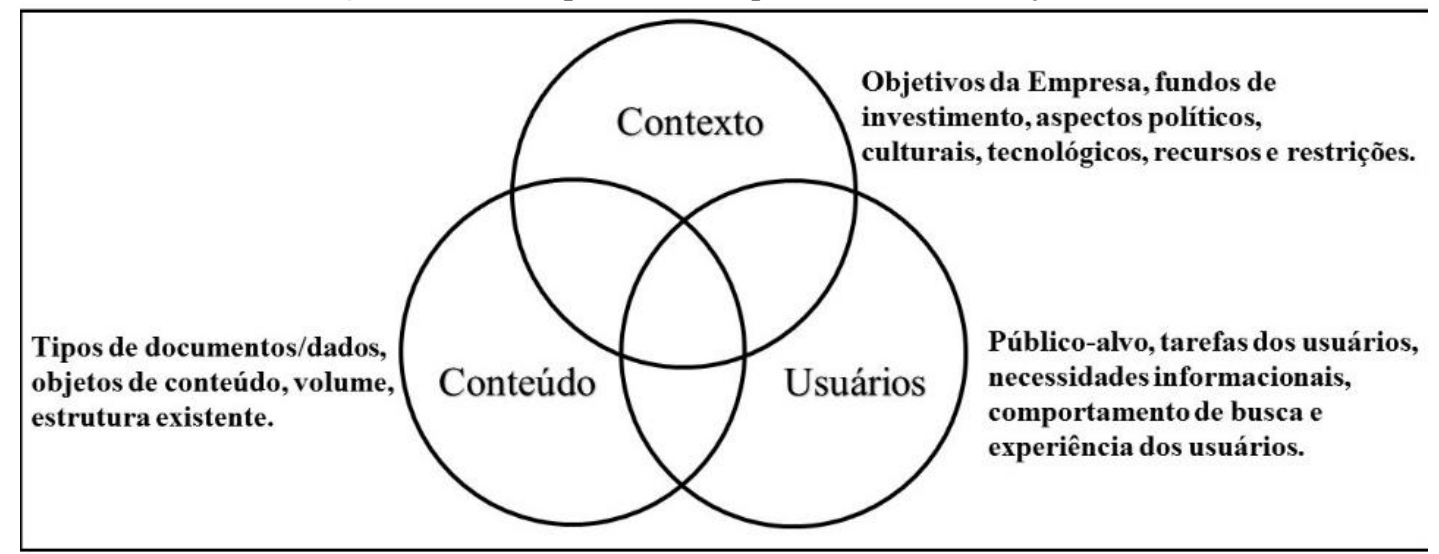

Fonte: Adaptado de Morville e Rosenfeld (2006)

Com base em Barker (2005) e Morville e Rosenfeld (2006), o contexto está relacionado à compreensão dos objetivos de uma organização (empresa/instituição), aspectos políticos, culturais, tecnológicos, recursos e limitações. Na prática, todo o contexto de uma instituição deve ser levado em 
consideração durante o desenvolvimento de um projeto de Arquitetura da Informação. A quantidade e qualidade do conteúdo são importantes na construção do projeto informacional, bem como a propriedade do conteúdo, seu formato, sua estrutura, seus metadados, seu volume de informações e seu dinamismo. Para que a AI seja eficaz, ela deve refletir-se na forma com que o sistema interage com o pensamento dos usuários e, deste modo, estudar o comportamento das pessoas e conhecer como utilizam o sistema, quem o utiliza e o que elas estão procurando, aspectos fundamentais para se ter êxito durante a elaboração do projeto.

Garret (2011) apresenta cinco elementos relacionados à experiência do usuário na web. O primeiro elemento é a estratégia, a necessidade de entender os motivos pelos quais as pessoas que navegam no website querem sair dele, antepondo, a uma saída aleatória, a finalização da tarefa de maneira satisfatória. O escopo do site, segundo elemento, é a etapa onde suas características e funções são definidas para a conclusão da estrutura do site, que, correspondente ao terceiro elemento. Durante a estruturação, se define como os usuários têm acesso à página e para onde eles poderiam ir quando concluírem a navegação. $\mathrm{O}$ quarto elemento é a projeção do "esqueleto" do site, composto por botões, fotos e blocos de texto. O esqueleto é desenvolvido com o propósito de aperfeiçoar a organização desses elementos de moro a torná-los mais eficientes. Por fim, o último elemento a ser elaborado é a interface do website, composta por imagens e textos.

Percebe-se que a Arquitetura da Informação tem estudos dirigidos ao ambiente da web por conta de sua importância para o aprimoramento de websites e demais aspectos relacionados. No presente estudo, consideramos a realização pesquisas na web como base científica para aplicação dos princípios da AI nos dispositivos móveis.

\subsection{Uso da Arquitetura da Informação nos dispositivos móveis}

Os dispositivos móveis possuem características diferentes das apresentadas por um computador, incluindo seu tamanho, resolução e orientação de tela, além dos 
métodos de entrada. Morville e Rosenfeld (2006) apresentam os sistemas de organização, rotulagem, busca e navegação em páginas da web, todos passíveis de serem utilizados para projetos que envolvem a aplicação em dispositivos móveis, mesmo com diferenças peculiares entre os aparelhos. Busca-se, desta forma, aproximar os sistemas apresentados pelos referidos autores aos dispositivos móveis:

a) sistema de organização - pode ser desenvolvido com base em esquemas exatos de organização da informação ou esquemas estruturantes da informação. A utilização de ordem alfabética, cronológica ou geográfica são possibilidades que podem ser aplicadas nos dispositivos móveis, sendo fundamental estabelecer hierarquias e evitar ambiguidade nesses sistemas, tendo em vista que os usuários estão cada vez mais objetivos e impacientes, esperando encontrar a informação que desejam da forma mais prática e simples possível e em um curto espaço de tempo. Assim, as informações principais devem estar à disposição na tela inicial do dispositivo móvel sem que haja a necessidade do usuário procurar através da navegação. Um sistema de organização bem definido proporciona uma recuperação de informação eficiente: a organização por tópicos, divididas por assuntos ou por área de interesse é uma alternativa a ser utilizada, pois além de direcionar tarefas de acordo com cada usuário e permitir seu uso a partir das suas escolhas, trabalhar com páginas personalizadas de acordo com suas preferências. Ademais, ao estruturar a informação para os dispositivos móveis, há a necessidade de se utilizar esquemas hierárquicos, bem como taxonomias apropriadas;

b) sistema de rotulagem - o motivo pelo qual a aplicação de rótulos nos dispositivos móveis constitui uma tendência atual é devido ao tamanho reduzido das telas, que dificultam a inclusão de textos. Geralmente, utilizam-se os rótulos para facilitar a navegação do usuário, substituindo palavras e menus textuais por ícones que representam alguma informação. Os rótulos devem ser familiares e tradicionalmente utilizados para que o usuário não precise inferir seus significados e, 
portanto, tome suas decisões no menor espaço de tempo possível. Além disso, menus convencionais que associam ícones, textos e links podem ser substituídos por apenas ícones com links para facilitar a navegação dos usuários. É imprescindível que o planejamento dos rótulos desenvolvidos para dispositivos móveis seja feito levando em consideração as características de cada aparelho, como seu tamanho de tela e forma de navegação;

c) sistema de navegação - geralmente são complexos devido à variedade de elementos básicos, também conhecidos como subsistemas. Deve-se levar em consideração os aspectos globais e locais da navegação, apresentar, de forma clara e consistente, uma estrutura hierárquica de informações, indicando a localização atual do usuário e para onde ele pode navegar a partir dali. Um sistema de navegação geral deve estar contido em todas as páginas de forma a permitir o acesso direto para as principais áreas e funções. Assim, levando em conta as características dos dispositivos móveis, faz-se necessário um planejamento estrutural das páginas que contemple todo o sistema de navegação e adéque-o ao tamanho de tela e modo de navegação de cada aparelho;

d) sistema de busca - está diretamente associado à eficiência da recuperação de informação realizada pelos usuários. O sistema de busca é mais complexo do que a ferramenta de busca, devendo contemplar aspectos como: o questionamento do usuário ou os termos de busca utilizados na pesquisa; a interface da busca através das linguagens e construtores da consulta; o motor de busca, bem como o conteúdo (vocabulários controlados e metadados); e, os resultados apresentados através do agrupamento, disposição por ordem de relevância ou outras características definidas por algoritmos. É recomendado que, nos dispositivos móveis, haja um sistema de busca simples que liste os resultados por tópicos e utilize imagens ou ícones para facilitar a assimilação do usuário. 
Firtman (2013) comenta que a estratégia utilizada nos dispositivos móveis deve oferecer aos usuários a liberdade de escolha ao navegar. $\mathrm{O}$ arquiteto da informação pode projetar aplicativos ou desenvolver páginas adaptadas às características dos aparelhos, devendo fazê-lo com base na estratégia da instituição para qual preste serviço e, principalmente, de acordo com o contexto em que seu trabalho será inserido, como: onde estão os usuários? O que os usuários procuram? O que posso desenvolver para ajudar a resolver os problemas dos usuários? Onde costumam acessar o dispositivo móvel, no trabalho, na rua, viajando, assistindo TV? Dentre outros questionamentos referentes ao público em questão.

Os projetos de Arquitetura de Informação para dispositivos móveis devem seguir o princípio da melhoria contínua, apresentado por Firtman (2013), que inclui: promover conteúdo básico e acessível a todos os tipos de aparelhos; proporcionar funcionalidade básica e acessível a todos os navegadores; contemplar a semântica em todo conteúdo; oferecer layout aprimorado através do CSS (guia de estilos) vinculado externamente; utilizar o JavaScript para aprimoramento do comportamento dos usuários; e respeitar as preferências de navegador adotadas por eles. Em relação à navegação, o autor recomenda:

a) definir e ordenar as tarefas mais utilizadas pelos usuários através das informações coletadas ou de testes de usabilidade;

b) manter a consistência visual com o site convencional;

c) permitir aos usuários a realização de uma atividade em no máximo três toques;

d) limitar, em cinco, o número de seções abaixo da página inicial;

e) oferecer aos usuários a possibilidade de utilizar o site convencional através de um link denominado "versão clássica";

f) localizar os usuários se for útil para a instituição;

g) reduzir o número de formulários e evitar que os usuários tenham a necessidade de inserir muitos textos, tendo em vista a dificuldade de digitação causada pelo tamanho reduzido da tela; 
h) evitar o uso de páginas de boas-vindas em aplicativos baseados no navegador;

i) prever a navegação dos usuários através do contexto e histórico de navegação a fim de reduzir o número de páginas selecionadas e de cliques;

j) oferecer mecanismos de navegação, como o botão "voltar", independente do navegador escolhido pelos usuários.

Um exemplo de diferença entre a navegação em um dispositivo móvel e a convencional, adotada em um desktop, refere-se a disposição do conteúdo que, levando em consideração o visor pequeno, não deve ser divido em duas ou três colunas, como acontece normalmente em websites onde a navegação é feita por meio do computador. No Quadro 3, são apresentadas algumas recomendações que podem ser adotadas visando a experiência dos usuários.

Quadro 3 - Recomendações para dispositivos móveis

\begin{tabular}{|c|l|}
\hline $\mathrm{N}$ & Recomendações \\
\hline 1 & Evitar a rolagem horizontal \\
\hline 2 & $\begin{array}{l}\text { Utilizar a Web Responsive Design para promover o melhor projeto possível em } \\
\text { diferentes cenários, como as mudanças de orientação ou tamanho da tela }\end{array}$ \\
\hline 3 & Reduzir a quantidade de textos \\
\hline 4 & Utilizar fontes legíveis em todo tipo de tela e aparelho \\
\hline 5 & Utilizar cores para separar as seções \\
\hline 6 & Manter o número total de links em no máximo 15 por página \\
\hline 7 & Fornecer o link "ir para o topo" no rodapé \\
\hline 8 & Utilizar todo o espaço da tela para o conteúdo \\
\hline 9 & Fornecer os recursos mais utilizados pelos usuários no topo da tela \\
\hline 10 & Minimizar a quantidade de entrada de textos necessária por parte dos usuários \\
\hline 11 & $\begin{array}{l}\text { Preservar o histórico e configurações dos usuários com a finalidade de prever suas } \\
\text { ações }\end{array}$ \\
\hline 12 & $\begin{array}{l}\text { Utilizar cores que favoreçam a navegação do usuário em qualquer ambiente que esteja, } \\
\text { seja um lugar com má iluminação, nos transportes públicos ou na praia à luz do sol }\end{array}$ \\
\hline 13 & Fornecer diferentes estilos para dispositivos sensíveis ao toque \\
\hline 14 & Utilizar listas em vez de tabelas \\
\hline 15 & Oferecer itens de acessibilidade \\
\hline 16 & $\begin{array}{l}\text { Proporcionar a melhor experiência possível, independentemente do aparelho que o } \\
\text { usuário estiver utilizando }\end{array}$ \\
\hline 17 & Utilizar imagens coloridas em alta definição \\
\hline 18 & Ocultar a barra de URL para que se tenha mais espaço no app \\
\hline 19 & $\begin{array}{l}\text { Seguir padrões de navegação que são convencionais aos usuários em telas sensíveis ao } \\
\text { toque }\end{array}$ \\
\hline 20 & Fornecer feedback rápido quando o toque é aceito \\
\hline
\end{tabular}
Fonte: Adaptado de Firtman (2013) 
Os maiores desafios ao projetar uma Arquitetura da Informação nos dispositivos móveis estão relacionados com a redução do número de toques, pois os usuários de aparelhos como smartphones utilizam os dedos na navegação. Há também a necessidade de redução do conteúdo apresentado, devido à indisposição destes usuários em navegarem por muito tempo em ambientes com muitas informações. É recomendada a restrição de funcionalidades no projeto, por ela minimiza as chances de erros do usuário, que pode se confundir com um número excessivo de funções. Por fim, é fundamental oferecer-lhe a possibilidade de optar entre uso de páginas adaptadas à visualização em dispositivo móvel ou a versão convencional do website.

\subsection{O papel do arquiteto da informação nos dispositivos móveis}

Para Morrogh (2002), o profissional arquiteto da informação é um profissional de destaque e importância crescentes em uma economia em rede. O profissional atua no gerenciamento do projeto, desenvolvimento e implementação de informações em ambientes que possam tornar a experiência do usuário fácil e agradável. A função do arquiteto da informação é “[...] compreender o que está sendo desejado pela organização, devendo entender os negócios, seus objetivos e regras, além disso, carecem em ter conhecimentos multidisciplinares que estejam diretamente correlacionadas com suas funções [...]” (RIBEIRO; MONTEIRO, 2013, p. 792).

A multidisciplinaridade é uma característica inerente ao profissional da Arquitetura da Informação. Morrogh (2002) elenca sete áreas que o profissional deve ter conhecimento: Sistemas de informação/Gestão/Organização; Busca e recuperação de informação; Gerenciamento de banco de dados; Metodologia de Pesquisa/Estatística; Interação Humano-Computador; Rede de Computadores; e Gerenciamento de Projetos/Solução de Problemas.

A dificuldade de se trabalhar com dispositivos móveis pode ser considerada ainda maior devido à dinâmica tecnológica que sofrem os aparelhos, tornando-se obsoletos em pouco tempo e abrindo espaço para novos modelos que os substituirão. Além dos conhecimentos multidisciplinares, o 
profissional da arquitetura da informação deve ter a capacidade de se adequar às mudanças e, principalmente, de projetar pensando em um futuro, sempre tendo em mente o usuário.

As características defendidas por Wurman (1996) refletem as atuais capacidades que o profissional deve possuir; o autor defende que o arquiteto da informação deve criar estruturas e planejar informações de modo a conduzir os usuários até o caminho mais simples e objetivo à sua necessidade. Portanto, o papel do profissional da Arquitetura da Informação deve ser mantido, porém adaptado às novas características apresentadas pelos dispositivos móveis.

\section{Interação homem-dispositivo móvel e sua aplicação nas unidades informacionais}

A interação que sempre ocorreu nas relações humanas, ocorre também com as tecnologias de informação. Na literatura, o termo mais conhecido que trata da relação entre o homem e a máquina é o IHC - Interação humano-computador ou, em inglês, HCI - human-computer interaction. A princípio, o surgimento do termo tinha o objetivo de investigar sobre novos tipos de tecnologias de informação e suas relações de interação com os usuários. Com o avanço tecnológico, o foco continua o mesmo, porém com um artefato diferente - o dispositivo móvel.

Rocha e Baranauskas (2003) afirmam que os principais objetivos do IHC são: produzir sistemas acessíveis, seguros e funcionais; desenvolver ou melhorar a segurança, utilidade, efetividade e usabilidade de sistemas computacionais. Já Nielsen (1993) afirma que existem cinco fatores humanos que precisam ser analisados no momento de promoção da interação entre homem e máquina:

a) o tempo de aprendizado, que diz respeito a quanto tempo o usuário leva para aprender a usar os comandos relevantes para um conjunto de tarefas;

b) a eficiência de uso, que está relacionada ao tempo que o usuário utiliza para realizar uma determinada tarefa; 
c) a taxa de erros por usuários, relacionada aos erros que pessoas cometem com maior frequência;

d) a memorização, que está relacionada ao nível com o qual os usuários mantém os conhecimentos adquiridos após o uso;

e) a satisfação subjetiva, que trata de como os usuários gostam de utilizar os vários aspectos do sistema.

A interação entre homem e os dispositivos móveis contemplam os itens elencados por e Nielsen (1993) e Rocha e Baranauskas (2003), mas o que a difere do convencional é a ampliação da interatividade, ao passo que os dispositivos móveis passaram a fazer parte do cotidiano das pessoas e a representarem uma importante ferramenta em muitas de suas atividades, de pessoais à profissionais. Aparelhos com telas sensíveis ao toque, reconhecimento de voz, teclado com correção de texto automática e telas que reconhecem a aproximação dos dedos são exemplos da ampliação dessa interatividade. O que adotamos - Interação homem dispositivo-móvel - surge como uma proposta de utilização e de discussão para pesquisas futuras. Pretende-se, desta forma, apresentar a ampliação da interação decorrente da facilidade e disponibilidade de uso dos aparelhos móveis para os seres humanos.

Como o acesso aos dispositivos móveis está cada vez mais democrático, as unidades informacionais como as bibliotecas, arquivos e museus podem aproveitar a oportunidade para se adequarem às novas exigências dos usuários. A implementação de aplicativos desenvolvidos para dispositivos móveis nas unidades de informação aumenta a interatividade entre as unidades informacionais e os usuários, gerando benefícios como: disponibilização de conteúdo digital aos usuários; facilidade no processo de busca e recuperação da informação; comunicação e troca de informação entre os usuários conectados; consulta de informações online; feedback dos usuários em relação aos serviços prestados; e utilização de QR code, um código de barras que armazena links para transmitir informações aos aparelhos; e fóruns de discussão onli-ne. 
O profissional da CI, por sua vez, pode utilizar essa interação para aprimorar seus serviços e proporcionar novas experiências aos usuários, que, por exemplo, podem ter uma biblioteca no bolso e consultá-la a qualquer hora do dia, em qualquer lugar, por meio de um smartphone. Todavia, Souza, Torres e Amaral (2011) alertam que envolvimento e compromisso são necessários por parte dos profissionais e instituições que pretendem utilizar a tecnologia móvel para interagir com os usuários.

O desafio é desenvolver novas aplicações que supram as lacunas informacionais e tornem os usuários autônomos durante o uso de um dispositivo móvel. A liberdade é um fator chave no processo de interação entre o homem e a máquina, pois faz dos usuários seres ativos na relação com seus dispositivos móveis.

\section{Considerações finais}

Desde que foi utilizada pela primeira vez na década de 70 por Wurman, a Arquitetura da Informação ocupa lugar de importância no processo de gestão da informação. É notório como o uso dos princípios da AI em qualquer ambiente onde a informação precise ser gerida, traz benefícios aos usuários, instituições, profissionais da informação e demais partes envolvidas no projeto. No tocante à pesquisa, a proposta de aplicar os princípios da Arquitetura da Informação aos dispositivos móveis foi baseada na premissa de que esta pode ser utilizada em qualquer ambiente informacional digital. Sendo assim, conclui-se que é possível aplicar os sistemas de organização, rotulagem, navegação e busca respeitando os limites e diferenças de cada aparelho, seja ele um smartphone, tablet ou ereader.

Durante a elaboração de um projeto de aplicação aos dispositivos móveis, as premissas desenvolvidas e os princípios estabelecidos sobre a Arquitetura da Informação devem ser respeitados, observando à aplicabilidade para cada modelo de aparelho, de acordo com o tamanho da tela e suas configurações próprias. O uso da AI nos dispositivos gera oportunidades de utilização nas unidades informacionais, e, portanto, deve ser gerido por 
profissionais da informação capacitados para conduzir o processo de implementação.

Os usuários estão, cada vez mais, utilizando dispositivos móveis em seu cotidiano, o que por sua vez fomenta o advento de aparelhos com maior interatividade que facilitam a realização de tarefas do dia-a-dia. Diante desse cenário e por meio da ampliação da interatividade proporcionada por esses dispositivos, as unidades informacionais devem se adequar à nova realidade, investindo em pesquisa acerca do desenvolvimento ou uso de aplicativos para o aprimoramento de seus serviços online, a exemplo das bibliotecas que interagem com seus usuários digitalmente, oferecendo atendimento $24 \mathrm{~h}$ por dia. Antes, porém, é recomendado ouvir os usuários a fim de compreender suas necessidades e, assim, poder promover-lhes uma experiência mais agradável através dos princípios da Arquitetura da Informação.

A relevância deste tipo de estudo para a Ciência da Informação está diretamente relacionada tanto à carência de pesquisas com a temática quanto ao fato das novas plataformas tecnológicas, como os dispositivos móveis, poderem ser utilizados nas unidades informacionais. Desta forma, recomenda-se aos profissionais da informação que se capacitem continuamente para melhor compreenderem os aspectos técnicos dos dispositivos móveis, para entender o comportamento dos usuários destes aparelhos e para estudar sobre o modo como a Arquitetura da Informação pode ser implementada nos dispositivos móveis.

\section{Referências}

AMOROSO, D. Leitores digitais: conheça mais sobre esses aparelhos. 2009. Disponível em: <http://www.tecmundo.com.br/2557-leitores-digitais-conhecamais-sobre-esses-aparelhos.htm>. Acesso em: 22 nov. 2014.

ALBUQUERQUE, A. R. R. de. Discurso sobre fundamentos de arquitetura da informação.2010. 241 f. Tese (Doutorado)-Faculdade de Ciência da Informação, Universidade de Brasília, Brasília, 2010.

ARAÚJO, C. A. A. O que é Ciência da Informação? Informação \&

Informação, Londrina, v. 19, n. 1, p. 1-30, jan./abr. 2014. 
BARKER, I. What is information architecture.2005. Disponível em: <http://www.steptwo.com.au/papers/kmc_whatisinfoarch>. Acesso em: 10 nov. 2014.

BAYLE, S. Information architecture: a brief introduction.2003. Disponível em: 〈http://aifia.org/tools/download/Bailey-IAIntro.pdf>. Acesso em: 11 mai. 2015.

COSTA, I. de M. Um método para arquitetura da informação:

fenomenologia como base para o desenvolvimento de arquiteturas da informação aplicadas. 2010. 149f. Dissertação (Mestrado) - Faculdade de Ciência da Informação, Universidade de Brasília, Brasília, 2010.

DILLON, A. Information architecture in JASIST? Journal of the American Society for Information Science and Technology, North Carolina, v. 53, n.10, p. 821-823, ago. 2002.

DUARTE, J. C. Uma arquitetura ágil da informação organizacional. 2010. 210 f. Tese (Doutorado em Ciência da Informação) - Faculdade de Ciência da Informação, Universidade de Brasília, Brasília, 2010.

EWING, C.; MAGNUSON, E.; SCHANG, S. Information architecture proposed curriculum. Austin: UTIAG, 2002. Disponível em:

$\langle$ http://www.gslis.utexas.edu/ iag/resources/ia-curriculum-final.PDF>. Acesso em: 20 nov. 2014.

FIRTMAN, M. Programming the mobile web. 2. ed. Sebastopol: O'Reilly Media, 2013.

FITZEK, F.; REICHART, F. Mobile phone programming: and it's application to wireless network. Dordrecht: Springer, 2007.

GARRET, J. J. The elements of user experience: user centered design for the web and beyond. 2. ed. Berkeley, CA: News Riders, 2011.

HAGEDORN, K. The information architecture glossary. [S. n]: ACIA, 2000. Disponível em: 〈http://argus-acia.com/white_papers/iaglossary.html〉. Acesso em: 11 maio 2015.

HAVERTY, M. Information architecture without internal theory: an inductive design process. Journal of the American Society for Information Science and Technology, North Carolina, v. 10, n. 53, p. 839-845, 2002.

INFORMATION ARCHITECTURE INSTITUTE. What is Information Architecture? 2013. Disponível em:

<http://iainstitute.org/documents/learn/What_is_IA.pdf>Acesso em: 20 nov. 2014. 
LACERDA, F. Arquitetura da informação: aspectos epistemológicos, científicos e práticos. 2005. 186 f. Dissertação (Mestrado) - Faculdade de Ciência da Informação, Universidade de Brasília, Brasília, 2005.

LIMA MARQUES, M. Outline of a theoretical framework of architecture of information: a School of Brasilia proposal. In: BÉZIAU, J.; CONIGLIO, M. E. (Ed.). Logic without Frontiers: festschrift for Walter Alexandre Carnielli on the occasion of his 60th birthday. London: College Publications, 2011. (Tribute Series, v. 17). p. 311-320.

MORROGH, E. Information architecture: an emerging 21st century profession. New Jersey: Prentice Hall, 2002. Disponível em: $\langle$ http://iainstitute.org/documents/learn/education/morroghchapter21.pdf>. Acesso em: 26 nov. 2014.

MORVILLE, P.; ROSENFELD, L. Information architecture for the World Wide Web. Sebastopol: O’Reilly Media, 1998.

MORVILLE, P.; ROSENFELD, L. Information architecture for the World Wide Web: designing large-scale web sites. 3. ed. Sebastopol: O’Reilly Media, 2006.

NIELSEN, J. Usability engineering. Boston: Academic Press, 1993.

OLIVEIRA, C. B. Uma proposta de arquitetura da informação para o processo de inovação em centros de pesquisa. 2012. 235 f. Tese (Doutorado) - Faculdade de Ciência da Informação, Universidade de Brasília, Brasília, DF, 2012.

PINTO, R. A história dos tablets: descubra como tudo começou. 2014. Disponível em: <http://informaticasimples.net/historia-dos-tablets-descubracomo-tudo-comecou>. Acesso em 22 nov. 2014.

RENATO, F. A história dos telefones celulares. 2012. Disponível em: <http://www.techtudo.com.br/artigos/noticia/2012/06/historia-dos-telefonescelulares.html >. Acesso em: 22 nov. 2014.

REISS, E. L. Practical information architecture: a hands-on approach to structuring successful sites. New York:Addison Wesley, 2000.

RESMINI, A; ROSATI, L. Pervasive Information Architecture: designing cross-channel user experiences. Burlington: Elsevier, 2011.

RIBEIRO, F. F.; MONTEIRO, S, D. O profissional arquiteto da informação. In:SEMINÁRIO EM CIÊNCIA DA INFORMAÇÃO, 5., 2013, Londrina. Anais eletrônicos...Londrina: UEL, 2013. Disponível em: <http://www.uel.br/grupopesquisa/ciberespaco/doc/E5_RIBEIRO.pdf>. Acesso em: 26 nov. 2014. P. 785801. 
ROCHA, H. V.; BARANAUSKAS, M. C. C. Design e avaliação de interface humano-computador. São Paulo: UNICAMP, 2003.

SCHILTZ, M.; TRUYEN, F.; COPPENS, H. Cutting the trees of knowledge: social software, information architecture and their epistemic consequences. Thesis Eleven, [S.1.], n. 89, p. 94-114, May 2007.

SILVA, Z; SOUZA, E. A produção interdisciplinar na ciência da informação: indicadores dos fundamentos na arquitetura da informação. In: ENCONTRO NACIONAL DE PESQUSIA EM CIÊNCIA DA INFORMAÇÃO, 15., 2014, p. 319-326, Belo Horizonte. Anais eletrônicos...Belo Horizonte: ANCIB, 2014. Disponível em: 〈http://enancib2014.eci.ufmg.br/documentos/anais/anais-gt1> Acesso em: 22 nov. 2014.

SIQUEIRA, A. H. Arquitetura da informação: Uma proposta para fundamentação e caracterização da disciplina científica. 2012. 402 f. Tese (Doutorado em Ciência da Informação) - Faculdade de Ciência da Informação, Universidade de Brasília, Brasília, 2012. Disponível em:

<http://repositorio.unb.br/handle/10482/12157?mode=full>. Acesso em: 11 maio 2015.

SIQUEIRA, E. Mundo terá 55 bilhões de dispositivos móveis em 2020. 2011. Disponível em: <http://blogs.estadao.com.br/ethevaldo-siqueira/2011/02/21/55bilhoes-de-dispositivos-moveis/>. Acesso em 22 nov. 2014.

SOUZA, M. I. F; TORRES, T. Z; AMARAL, S. F. Bibliotecas digitais e dispositivos móveis: acesso a novos espaços de aprendizagem. In:

CONGRESSO BRASILEIRO DE BIBLIOTECONOMIA, DOCUMENTAÇÃO E CIÊNCIA DA INFORMAÇÃO, 24., 2011, Maceió. Anais... Maceió: FEBAB, 2011. Disponível em: $<$ http://www.alice.cnptia.embrapa.br/alice/bitstream/doc/898118/1/Bibliotec asdigitais.pdf>. Acesso em: 29 set. 2015.

WURMAN, R. S; BRADFORD, P. Information architects. Zurich: Graphis Press, 1996.

\title{
Reflections about information architecture for mobile devices
}

\begin{abstract}
The article presents an analysis about the application of the principles of Information Architecture (IA) to mobile devices through the concept and technological evolution of these devices and their adequacy according to the recommendations made by Morville and Rosenfeld (2006) and Firtman (2013),
\end{abstract}


in addition to presenting the interaction expansion between human and mobile devices. The adopted methodology was an exploratory search that investigates the adoption of the principles of IA to mobile devices through a literature review. We conclude that despite the inherent characteristics of mobile devices, it is possible to apply the systems of organization, labeling, navigation, and search while also regarding the limitations and differences of each device, whether it is a smartphone, tablet or e-reader. The interaction be-tween man and mobile devices has been extended due to the available technological resources and the increase in time spent by users utilizing and navigating these mobile devices. It is been recommended for information professionals to adapt to this new reality and to develop applications based on the information architecture with the intent to promote an enjoyable and satisfactory experience for users, especially in the informational units.

Keywords: Information Architecture. Mobile Devices. Human-Mobile Devices Interaction. Digi-tal Information Environments.

Recebido: 14/05/2015

Aceito: 20/10/2015 